\title{
Recent Results of the Auger Engineering Radio Array (AERA)
}

\section{Ewa M. Holt ${ }^{* a, b}$ for the Pierre Auger Collaboration ${ }^{c}$}

${ }^{a}$ Karlsruhe Institute of Technology - KIT, Institut für Kernphysik, 76021 Karlsruhe, Germany

${ }^{b}$ Instituto de Tecnologías en Detección y Astropartículas (CNEA, CONICET, UNSAM), Buenos

Aires, Argentina

${ }^{c}$ Observatorio Pierre Auger, Av. San Martín Norte 304, 5613 Malargüe, Argentina

E-mail: auger_spokespersons@fnal.gov

Full author list: http://www.auger.org/archive/authors_icrc_2017.html

The Auger Engineering Radio Array (AERA) is located at the Pierre Auger Observatory in Mendoza Province, Argentina. More than 150 autonomous antenna stations, spread over $17 \mathrm{~km}^{2}$, are used to measure the radio emission from extensive air showers initiated by cosmic rays with energies above $0.1 \mathrm{EeV}$ in the frequency range of $30-80 \mathrm{MHz}$. AERA is operated in coincidence with the other detectors of the Observatory, which comprise an array of water-Cherenkov detectors, fluorescence telescopes and buried scintillators that are used to detect muons. This gives a unique opportunity of cross-calibration between the detector types and complementary analyses of shower parameters. From the radio measurements we reconstruct cosmic-ray properties like energy, arrival direction and estimators of the mass composition, in particular the atmospheric depth $X_{\max }$ of maximum shower development. To determine $X_{\max }$, we follow a top-down approach with detailed simulations down to the individual particle level, also considering the refractive index. Two independent implementations are followed, both using an atmospheric model based on GDAS data. The results of the two analyses are consistent. We achieve a resolution of the radio detectors of $\sim 39 \mathrm{~g} / \mathrm{cm}^{2}$. Since radio measurements are solely sensitive to the electromagnetic part of the shower, we can measure the electron-muon ratio in combination with the muon detector of AMIGA as an additional mass estimator. Recently AERA demonstrated that for inclined showers the area of the radio-emission footprint extends to several square kilometers. This shows great potential for future large-scale radio arrays, since the large footprints allow for a wide spacing of the antennas.

35th International Cosmic Ray Conference - ICRC2017

10-20 July, 2017

Bexco, Busan, Korea

* Speaker. 


\section{Introduction}

Over the last decade, the detection of air-shower radio emission has evolved from small-scale prototype setups to large-scale experiments performing measurements for cosmic-ray physics $[1,2]$. Radio detectors are ideal to be used in combination with other cosmic-ray detection techniques like particle, fluorescence and air-Cherenkov detectors, which enable cross-calibration between the detectors. Since the radio emission is solely produced by the electromagnetic part of the shower, its detection delivers complementary information to the measurements of all-particle and muon detectors. The radio technique has an advantage over the fluorescence and air-Cherenkov technique, since it has an operational time of nearly $100 \%$. It is only disturbed from high atmospheric electric fields which occur in massive rain clouds and during thunderstorms [3, 4]. In addition, the radiation does not get attenuated since the atmosphere is transparent for radiation in the $\mathrm{MHz}$ range. The radio emission is sensitive to the shower development, i.e., to the depth of the shower maximum $X_{\max }$, which is statistically related to the mass of the primary particle. Furthermore, the emission contains information about the arrival direction and energy $[5,6]$ of the cosmic ray.

\section{The Auger Engineering Radio Array (AERA)}

The Auger Engineering Radio Array is located at the Pierre Auger Observatory in Mendoza Province, Argentina [7]. It is part of the low-energy enhancements of the Observatory together with AMIGA [8] and HEAT [9]. AMIGA is a combination of water-Cherenkov detectors spread on a grid of $750 \mathrm{~m}$ spacing and buried scintillators at $2.3 \mathrm{~m}$ depth to solely measure the muons of the showers. The half spacing compared to the Surface Detector (SD) array in the rest of the Observatory lowers the energy threshold. With HEAT, the Fluorescence Detector (FD) is extended by three high-elevation telescopes to observe low-energy air showers which evolve higher in the atmosphere. All four detector types are co-located in the same area of the Observatory and measure cosmic rays down to $\sim 10^{17} \mathrm{eV}$ in coincidence. This allows for cross-calibration and complementary measurements. In addition, the SD and FD serve as a trigger for AERA.

AERA comprises 153 autonomous radio stations spread over an area of $\sim 17 \mathrm{~km}^{2}$. The array was completed in March 2015 after three deployment phases. As an engineering array, it combines different hardware, e.g., electronics and communication systems, different spacing between the antennas and different trigger concepts (internal and external). The dense center of AERA contains 24 logarithmic periodic dipole antennas (LPDA) on a $144 \mathrm{~m}$ grid.The rest of the array is built of butterfly antennas with $250 \mathrm{~m}, 375 \mathrm{~m}$ as well as $750 \mathrm{~m}$ spacing, the latter to measure inclined air showers. In figure 1, a map of AERA together with the other co-located detectors is shown and the different antenna types and trigger systems are indicated. The two antenna arms of each station are aligned in east-west and north-south directions, respectively, and are sensitive to the radio emission in the frequency range of $30-80 \mathrm{MHz}$. The antenna response pattern is calibrated using an emitting source attached to an octocopter, resulting in an overall uncertainty of $9.4 \%$ on the amplitude [10]. For the time synchronization between the antennas, a beacon transmitter [11] at the FD site transmits well-defined sine waves. The nanosecond-level accuracy of this method was confirmed by independent measurements using radio pulses emitted by commercial airplanes [12]. 


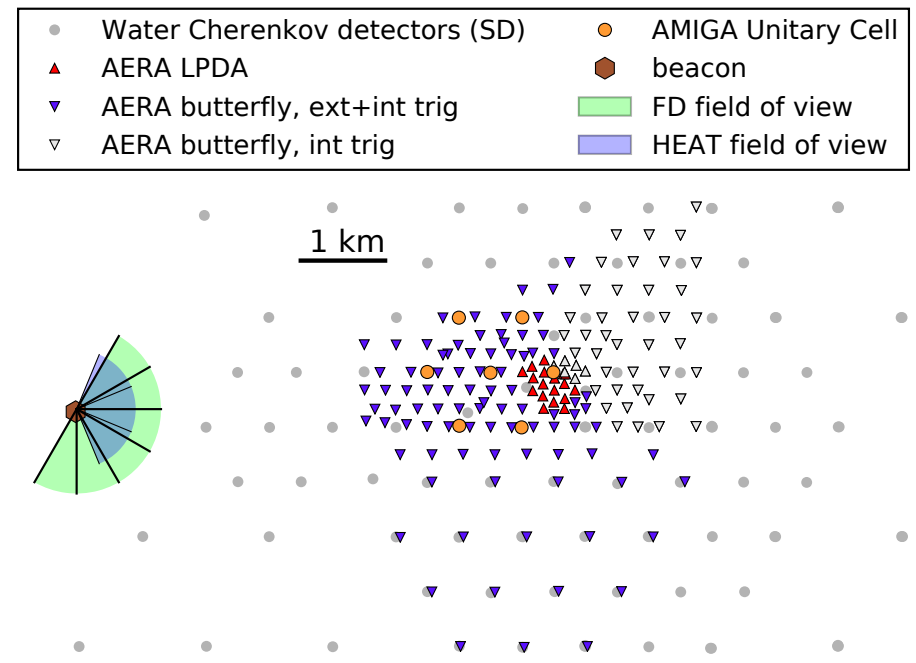

Figure 1: Map of the AERA array together with the other co-located detectors of the Pierre Auger Observatory and its enhancements.

\section{Radio $X_{\max }$ measurements}

The atmospheric depth at which the number of secondary particles in the shower reaches its maximum is called $X_{\max }$. It is statistically correlated to the mass of the primary cosmic ray since heavier particles interact higher in the atmosphere. The radio emission is mainly produced around $X_{\max }$ and emitted in a forward-directed cone around the direction of the propagation. Hence, the shape of the radio signal at the ground depends on the distance to the emission region and thus to $X_{\max }$. In AERA we follow several approaches to reconstruct $X_{\max }$ from the measured radio signal. The width of the 2-dimensional lateral distribution $[13,14]$ as well as shape parameters of the hyperbolic wavefront $[15,16]$ vary with the distance to $X_{\max }$. In addition, the spectral slope of the radio signals measured in the antennas is sensitive to $X_{\max }$ [17]. The best $X_{\max }$ resolution is obtained by evaluating the agreement of measured radio signals to air-shower simulations of different $X_{\max }$, as explained in the following.

The fundamental approach of this method was developed in [18] and adapted to the AERA detector in two independent analyses. The basic principle is to produce a set of Monte Carlo simulations of air showers with different $X_{\max }$ initiated by different primary particles, in particular protons and iron nuclei. The simulated radio signal is interpolated on a 2-dimensional map and fitted to the measured signals by a least $\chi^{2}$ fit. The minimum of the distribution of these least $\chi^{2}$ over their corresponding $X_{\max }$ gives the best fit for $X_{\max }$.

In the analysis of [14] (analysis A), a simulation set was produced for each measured event using the CoREAS code [19], the US standard atmospheric model and the measured direction and primary energy from the SD array. The set contained more proton simulations than iron to account for the higher shower-by-shower fluctuations of $X_{\max }$ for protons. The simulated electric field traces were transformed into energy fluences via the Poynting vector, which was then interpolated to a 2-dimensional lateral distribution. For each simulation the best fit for the shower core position in the AERA array was found with a least $\chi^{2}$ fit. The fit included a scaling factor to account for uncertainties in the input energy, absolute antenna calibration and simulated amplitude. A parabolic 

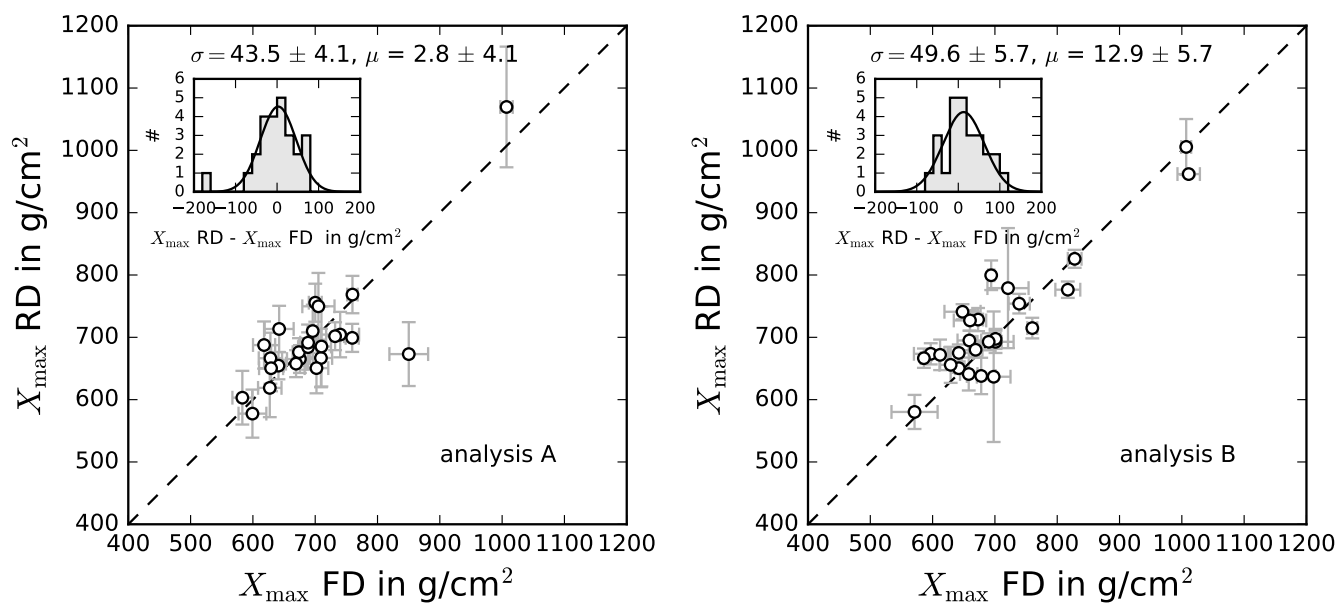

Figure 2: Reconstructed $X_{\max }$ from radio measurements based on simulated energy fluence distributions [14] (left, analysis A) and simulated amplitude distributions [22] (right, analysis B), compared to the measured FD $X_{\max }$. The dashed lines indicate a one-to-one correlation. The insets in both figures show the distributions of the differences together with a Gaussian fit.

function of the simulated $X_{\max }$ was fitted to the resulting distribution of least $\chi^{2}$ to find the best fit for $X_{\max }$. To take into account effects of atmospheric conditions at the time of the measurements, a correction factor based on GDAS data (Global Data Assimilation System) [20, 21] was applied on the $X_{\max }$ value at the end of the analysis chain. The method was applied to a high-quality data set of hybrid events measured from AERA (RD), SD and the fluorescence detector (FD) simultaneously. The results are compared to the reconstructed $X_{\max }$ values from the FD in the left panel of figure 2 .

A different, independent analysis with a similar approach was performed in [22] (analysis B). The simulations were calculated by the SELFAS code [23]. With GDAS data an air density and refractivity profile as a function of altitude were calculated for the time of the detected event and applied as atmospheric model in the simulations. The measured arrival direction of AERA and an arbitrarily chosen energy of $10^{18} \mathrm{eV}$ were taken as input, which makes the analysis independent of SD measurements. The simulated amplitude was fitted to the measured data by shifting the shower core and scaling the absolute amplitude. The scaling factor was used to determine the primary energy, since to first order the amplitude is linearly proportional to the energy. The minimum of the $\chi^{2}$ distribution determined the best fit value for $X_{\max }$. The analysis was applied to a high-quality set of hybrid events, using FD standard quality cuts and a minimum of 5 radio stations with signal. A comparison of the results to the FD $X_{\max }$ values is shown in the right panel of figure 2.

The radio $X_{\max }$ values of both analyses are compatible with the FD $X_{\max }$ values with a resolution of $44 \pm 4 \mathrm{~g} / \mathrm{cm}^{2}$ and $50 \pm 6 \mathrm{~g} / \mathrm{cm}^{2}$, respectively, and a small offset compared to the resolution. This results in an overall radio $X_{\max }$ resolution of about $39 \mathrm{~g} / \mathrm{cm}^{2}$ when subtracting the FD $X_{\max }$ resolution of $26 \mathrm{~g} / \mathrm{cm}^{2}$ at an energy of $10^{17.8} \mathrm{eV}$ [24]. The consistent results of the two independent AERA analyses confirm the validity of the method applied. Further investigations are needed to evaluate if the small differences in the results are caused by the different applications of the method or by the different simulation codes. A similar resolution of $\sim 40 \mathrm{~g} / \mathrm{cm}^{2}$ was accomplished with the sparse radio array of Tunka-Rex that determined the $X_{\max }$ resolution of the radio measurements by comparing to air-Cherenkov measurements [25]. With the dense core of LOFAR a resolution of 

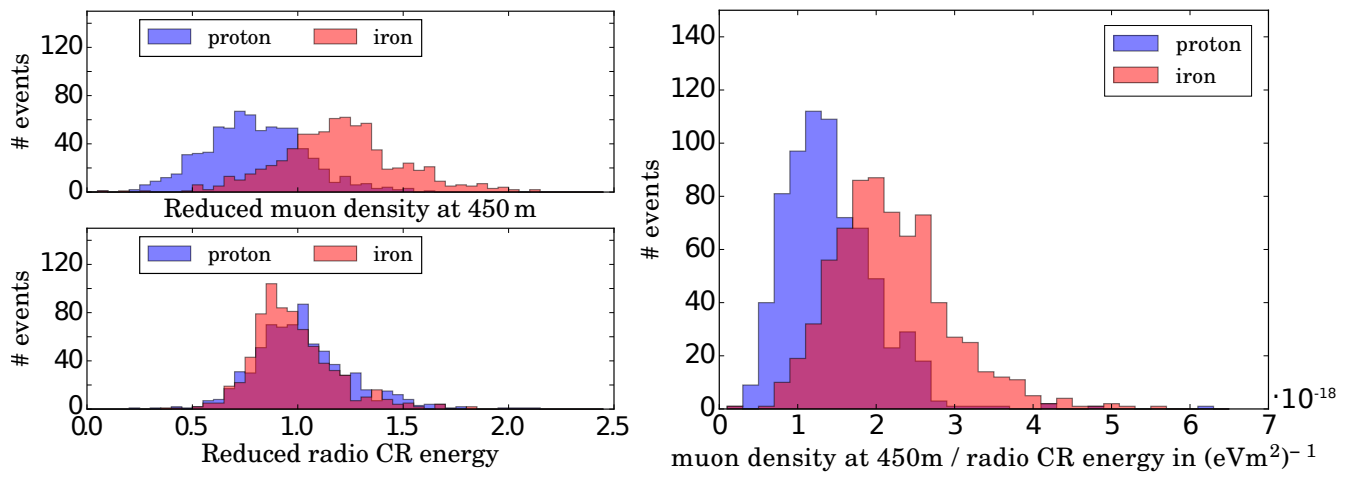

Figure 3: Simulations of hybrid measurements of radio emission and muons for proton- and iron-induced showers. Left upper panel: Muon density at a reference distance of $450 \mathrm{~m}$ from the shower axis relative to the mean muon density, reconstructed from the signal in the AMIGA scintillators. Left lower panel: Primary cosmic-ray energy reconstructed from the radio signal in the AERA antennas relative to the mean cosmic-ray energy. Right: Ratio of the muon density and primary cosmic-ray energy.

$17 \mathrm{~g} / \mathrm{cm}^{2}$ was gained [26].

\section{Hybrid measurements with muon detectors}

The water-Cherenkov measurement technique features only limited sensitivity to the type of the secondary particles arriving in the detectors. Instead, for air showers up to $\theta \leq 55^{\circ}$ separate measurements of the electromagnetic and muonic components are realized with the combination of AERA and AMIGA measurements [27]. The radio emission measured by AERA is solely produced by the charged electromagnetic part of the shower. The scintillators of AMIGA are buried at $2.3 \mathrm{~m}$ depth $\left(\approx 540 \mathrm{~g} / \mathrm{cm}^{2}\right.$ of vertical mass) to shield the electromagnetic part and solely measure the high-energy muons $(\mathrm{E}>1 \mathrm{GeV})$ of the showers at the ground. The relative magnitude of the electromagnetic and muonic components in the shower is a mass estimator complementary to $X_{\max }$. This concept has already been used, e.g., in the KASCADE-Grande experiment, where the ratio of the number of all charged particles to the number of muons at the ground was utilized to unfold the data in different elemental groups for a separate measurement of the cosmic-ray energy spectrum [28].

Contrary to particle measurements at the ground, the radio signal is produced along the shower development. To validate the mass sensitivity for the combination of the radio signal measured by AERA and the muon signal measured by AMIGA, air-shower simulations corresponding to measured AERA events for proton and iron primaries were studied. The amplitude and with it the total energy contained in the radio emission is correlated to the energy contained in the electromagnetic cascade, which induced this emission. A 2-dimensional double-Gaussian parametrization is used to fit the lateral distribution of the radio energy fluence (energy per unit area) at the ground $[13,5,6]$. The integral over this footprint yields the energy contained in the radio signal, the radiation energy. The primary cosmic-ray energy is reconstructed from the radiation energy for proton- and iron-induced air showers in the lower plot of the left panel of figure 3. It features on average $4 \%$ higher values for protons. The muon density at a reference distance of $450 \mathrm{~m}$ to the shower axis is correlated to the total number of muons at the ground and is reconstructed from the AMIGA scintillator data with a 

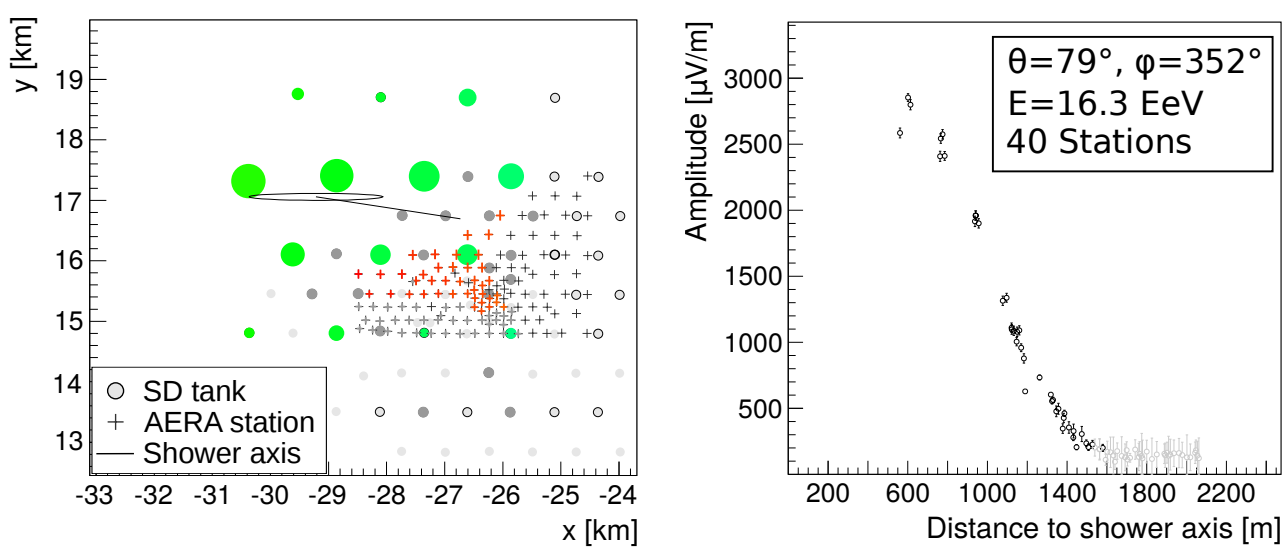

Figure 4: Example event of an inclined air shower with zenith angle of $\theta=79^{\circ}$. Left: AERA antenna stations and SD particle detectors with a signal. The sizes of the ' + ' and circles depict the signal strength in the radio antennas and the particle detectors, respectively. Grey stations are not taken into account in the analysis. Right: The lateral distribution of the radio signal with respect to the shower axis distance. Black and grey circles mark stations above and below the signal threshold, respectively. Even though the shower core is not contained inside of the AERA array, the shower is detected with a high resolution on the lateral distribution of the signal.

muon lateral-distribution function [8]. It is shown for proton- and iron-induced air showers in the upper plot of the left panel of figure 3, where the values are on average $40 \%$ higher for iron showers. The anti-correlated dependence on the primary mass maximizes the mass-separability for the ratio of the two observables, which is shown in the right panel of figure 3. Up to now, about 4 years of hybrid data of AERA and AMIGA are available to apply the findings in a combined analysis of this ratio.

The particle cascade of inclined air showers only contains muons when the shower arrives at the ground since the electromagnetic part is completely absorbed in the atmosphere. This leads to the opportunity of measuring the electromagnetic part with radio antennas and the muonic part with, e.g., water-Cherenkov detectors separately to determine the mass of the cosmic ray in future analyses.

\section{Radio emission from inclined air showers}

Since the radiation is beamed in the forward direction, the radio footprint on the ground has diameters of only a few hundred meters for near-vertical showers. This limits the maximum spacing between antennas for coincident measurements. The size of the footprint is almost independent of the primary energy, which is problematic for the detection of cosmic rays with the highest energies, where large detection areas are needed due to the low flux. However, simulations predict that the footprint grows to diameters of several kilometers for showers with zenith angles above $\sim 60^{\circ}$. This enables measurements of inclined air showers with sparse antenna arrays. For the first time, the lateral extent of such inclined air showers was measured with AERA [29, 30].

Since the shower maximum is geometrically more distant to the detector for higher zenith angles, the forward beamed emission is spread over a larger area. In addition, the large angle between the shower plane and the ground plane causes an elliptic cross-section of the signal at the 


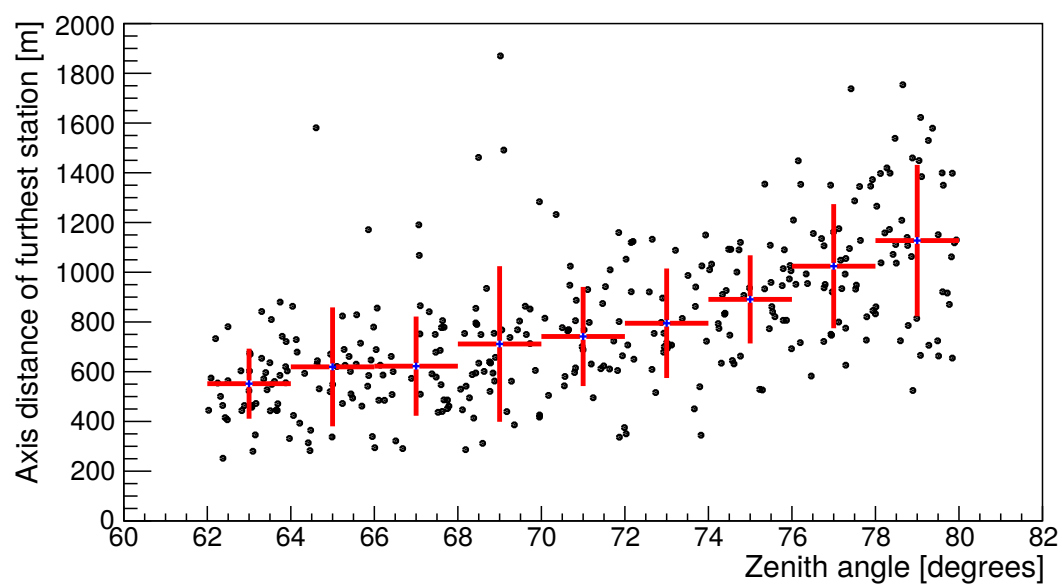

Figure 5: Distance of the furthest station with signal from the shower axis as a function of the zenith angle. The distances only depict a lower limit on the size of the radio footprints. Most footprints are not fully contained in AERA due to the limited size of the array.

ground. Hence, the total emission of the shower is spread over a larger area. However, this leads to a weaker signal in a single antenna and a higher energy threshold for the detection of inclined air showers.

The large area of AERA of $\sim 17 \mathrm{~km}^{2}$ and the co-location of water-Cherenkov detectors (SD) as a trigger, are ideal to measure such inclined air showers over the full extent of the footprint. A search for inclined air showers was performed with the 76 externally triggered antennas on the $144 \mathrm{~m}$ and $250 \mathrm{~m}$ grid (see figure 1). 344 events were measured in the zenith angle range between $62^{\circ}$ and $80^{\circ}$. An example event with a zenith angle of $79^{\circ}$ and 40 stations with a signal above background is shown in figure 4 . The high number of signal stations illustrates the large radio footprint of such a shower. In figure 5 the distance to the shower axis of the furthest radio station with signal above background is shown as a function of zenith angle for all 344 events. The furthest axis distance where the shower is detected rises with the zenith angle, which shows that the footprint is extended over larger areas for more inclined showers. However, the measured axis distances only constitute lower limits to the size of the footprints due to the insufficient size of AERA. In the future investigations with the 25 additional stations on a grid with $750 \mathrm{~m}$ spacing are planned to test the feasibility of a sparse array for the detection of inclined air showers.

\section{Conclusion}

AERA is dedicated to measuring the radio emission of cosmic-ray air showers above $10^{17} \mathrm{eV}$. It measures in hybrid mode with the other Auger detectors to gain complementary information for the reconstruction of the cosmic-ray properties. We reconstruct the shower maximum from various observables of the radio signal such as the size of the footprint, wavefront shape parameters, the spectral slope or by evaluating the best agreement to simulations. By comparing our results with the $X_{\max }$ measurements of the fluorescence telescopes we find a resolution of about $39 \mathrm{~g} / \mathrm{cm}^{2}$ with the latter method. We expect to improve the resolution with combining several methods in future analyses. Together with the buried scintillators we measure the electromagnetic and muonic components of the air shower separately. This allows us to measure the primary mass with the 
electron-muon ratio as a mass-sensitive parameter complementary to $X_{\max }$. For the first time, we measured the lateral extent of inclined air showers with the large area of AERA. We measured events with more than 70 signal stations with a distance to the shower axis of up to $2 \mathrm{~km}$.

\section{References}

[1] T. Huege, Phys. Rep. 620, 1 (2016)

[2] F. G. Schröder, Prog. Part. Nucl. Phys. 93, 1 (2017)

[3] W. D. Apel et al. [LOPES Collaboration], Adv. Space Res. 48, 1295 (2011)

[4] J. Neuser, PhD thesis, Bergische Universität Wuppertal (2015), URN:468-20160205-112223-7

[5] A. Aab et al. [Pierre Auger Collaboration], Phys. Rev. D 93, no. 12, 122005 (2016)

[6] A. Aab et al. [Pierre Auger Collaboration], Phys. Rev. Lett. 116, no. 24, 241101 (2016)

[7] A. Aab et al. [Pierre Auger Collaboration], Nucl. Instrum. Meth. A 798, 172 (2015)

[8] B. Wundheiler for the Pierre Auger Collaboration, 34th ICRC (2015), PoS(ICRC2015)324

[9] T. Hermann-Josef Mathes for the Pierre Auger Collaboration, 32nd ICRC 2011, Proc. 3, 153 (2011)

[10] A. Aab et al. [Pierre Auger Collaboration], Submitted to: JINST [arXiv:1702.01392].

[11] F. G. Schröder et al., Nucl. Instrum. Meth. A 615, 277 (2010)

[12] A. Aab et al. [Pierre Auger Collaboration], JINST 11, no. 01, P01018 (2016)

[13] A. Nelles, S. Buitink, H. Falcke, J. Hörandel, T. Huege and P. Schellart, Astropart. Phys. 60, 13 (2015)

[14] J. Schulz, PhD thesis, Radboud Universiteit Nijmegen (2016), http://hdl.handle.net/2066/151781

[15] Q. D. Hasankiadeh for the Pierre Auger Collaboration, 6th ARENA (2014), [arXiv:1705.06230]

[16] W. D. Apel et al., JCAP 1409, no. 09, 025 (2014)

[17] S. Grebe for the Pierre Auger Collaboration, 5th ARENA (2012), AIP Conf. Proc. 1535, 73-77 (2013)

[18] S. Buitink et al. Phys. Rev. D 90, no. 8, 082003 (2014)

[19] T. Huege, M. Ludwig and C. W. James, 5th ARENA (2012), AIP Conf. Proc. 1535, 128 (2013)

[20] NOAA Air Resources Laboratory (ARL), Global Data Assimilation System (GDAS1) Archive Information, Tech. rep. (2004). URL: http://ready.arl.noaa.gov/gdas1.php

[21] P. Abreu et al. [Pierre Auger Collaboration], Astropart. Phys. 35, 591 (2012)

[22] F. Gaté for the Pierre Auger Collaboration, Proc. XXV ECRS (2016), [arXiv:1702.02905]

[23] V. Marin and B. Revenu, Astropart. Phys. 35, 733 (2012)

[24] A. Aab et al. [Pierre Auger Collaboration], Phys. Rev. D 90, no. 12, 122005 (2014)

[25] P. A. Bezyazeekov et al. [Tunka-Rex Collaboration], JCAP 1601, no. 01, 052 (2016)

[26] S. Buitink et al., 7th ARENA (2016), EPJ Web Conf. 135, 01009 (2017).

[27] E. M. Holt for the Pierre Auger Collaboration, TAUP (2015), J. Phys. Conf. Ser. 718, no. 5, 052019 (2016)

[28] W. D. Apel et al., Astropart. Phys. 47, 54 (2013)

[29] O. Kambeitz, PhD thesis, Institut für Kernphysik, Karlsruhe Institute of Technology (2016), DOI:10.5445/IR/1000055758

[30] O. Kambeitz for the Pierre Auger Collaboration, 7th ARENA (2016), EPJ Web Conf. 135, 01015 (2017) 